\title{
Two-Dimensional Parameters Estimation
}

\author{
Shiv Gehlot \\ Department of Electronics \& Communication Engineering, Netaji Subash Institute of Technology, New-Delhi, India \\ Email: shivgehlot.nsit@gmail.com \\ Prof. Harish Parthasarathy \\ Department of Electronics \& Communication Engineering, Netaji Subash Institute of Technology, New-Delhi, India \\ Email: harishsignal@yahoo.com
}

\section{Ravendra Singh}

Department of Electronics \& Communication Engineering, Galgotias University, Greater Noida, India

Email: ravendra.singh@galgotiasuniversity.edu.in

\begin{abstract}
A parametric approach algorithm based on maximum likelihood estimation (MLE) method is proposed which can be exploited for high-resolution parameter estimation in the domain of signal processing applications. The array signal model turns out to be a superposition of two-dimensional sinusoids with the first component of each frequency doublet corresponding to the direction of the target and second component to the velocity. Numerical simulations are presented to illustrate the validity of the proposed algorithm and its various aspects. Also, the presented algorithm is compared with a subspace based technique, multiple signal classification (MUSIC) to highlight the key differences in performance under different circumstances. It is observed that the developed algorithm has satisfactory performance and is able to determine the direction of arrival (DOA) as well as the velocity of multiple moving targets and at the same time it performs better than MUSIC under correlated noise.
\end{abstract}

Index Terms - Direction of arrival, high resolution, two-dimensional, velocity estimation.

\section{INTRODUCTION}

The applications of high-resolution parameters estimation span a wide domain ranging from detection to surveillance. The two methods used to accomplish this are spectral based approach and parametric approach [1]. Speaking in the context of MUSIC, it is a high-resolution subspace based technique falling under the category of spectral based approach which utilizes covariance matrix to estimate the parameters of interest. MUSIC [2], [3] has a very high resolution and it assumes noise to be uncorrelated which leads to the diagonal correlation matrix. As a major drawback [4] MUSIC cannot be termed as a general approach as it requires advance information about the number of elements.

Estimation of Signal Parameter via Rotational Invariance Matrix (ESPRIT) [5] is also based on subspace-based approach and is a key contributor to the estimation theory. ESPRIT again is a high-resolution parameter estimation technique and has the additional advantage of lesser computational complexity. Its assumptions include sources to be centered at the same particular frequency and doublets of arrays that are not displaced rotationally [6], [7].

Parametric approach methods include Least Square Estimation (LSE) and Maximum Likelihood Estimation (MLE) [8], [9]. The maximum-likelihood method unlike least square method which models the estimation problem as a deterministic procedure demands probabilistic interpretation of all the interferences rather than the information of the parameters. Also, MLE output the parameter that is most likely responsible for producing data while LSE shortlists the parameter that seems to be the closest interpretation of the received data.

But, MLE estimates differ from LSE estimates when received data is not normally distributed and uncorrelated and different results are obtained depending upon the choice of the method between the two. However, in such a scenario MLE is preferred over LSE if probability distribution function description of the data is available. Also, both MLE and LSE arrive at same estimates if the data is normally distributed with constant variance [10], [11], [12].

In this paper, a MLE based algorithm is proposed for the estimation of different parameters of moving targets using electromagnetic sensor array. MLE suffers the disadvantage of higher computational complexity because it spans the entire signal space but has the side advantage of high-resolution parameter estimation even when the received data is corrupted by correlated noise, a condition under which MUSIC fails.

The rest of the paper is organized as follows. Section II deals with the problem formulation and derivation of the data model for the proposed algorithm. In section III, the developed data model is used for parameters estimation (DOA and velocity) using MLE. Section IV covers the simulations used for the verification of the algorithm. In section V, the MUSIC is discussed briefly and simulations are carried out to analyze the performance of the MUSIC under different circumstances with a motive of comparison 
between MUSIC and developed algorithm. Section VI discusses the Cramer- Rao bound, its role in the classification of the different estimators and Cramer-Rao bound calculation for the present algorithm. Section VII finally concludes the observations and results of the paper.

\section{DATA Model}

For the illustration of the proposed algorithm, the discussion to be considered here is focused on DOA and velocity estimation. In signal processing, for solving such a problem, the sensors array is deployed to collect the data from the radiating sources for estimating their different parameters. Few assumptions are further invoked for the analytical compliance of the problem. The radiations from the sources are assumed to be propagating in straight lines. A dispersive and isotropic transmission medium is required to validate the above assumption. Also, the radiations striking the array can be considered as plane waves if sources are within the far-field of the array. The narrowband signals $\left(s_{k}(t)\right)$ are assumed to have center frequency $\left(\omega_{o}\right)$ so that the general wave equation is given as:

$$
E(\mathbf{r}, t)=s(t) \operatorname{Re}\left[e^{j\left(\omega_{o} t-\mathbf{k} . \mathbf{r}\right)}\right]
$$

where $\mathbf{k}$ is propagation vector and $\mathbf{r}$ is position vector.

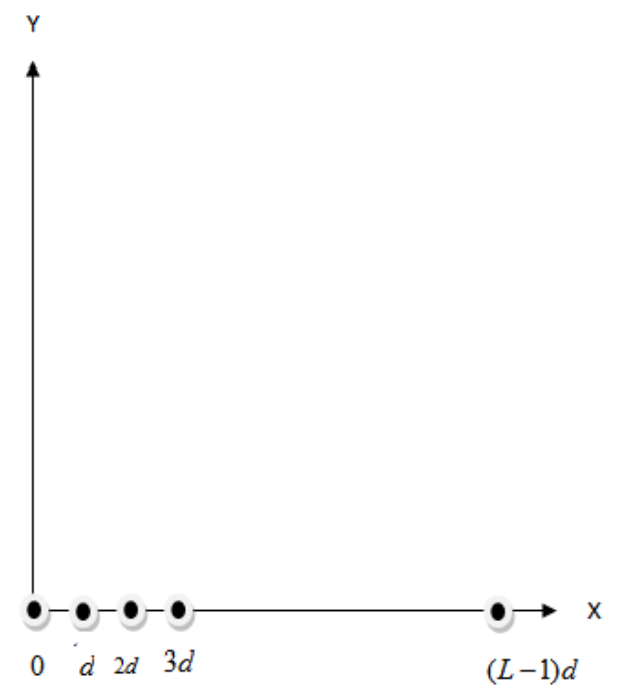

Fig.1. Uniform linear array

If we have a uniform linear array (ULA) [13] having $L$ sensors and separation $d$ between them (Fig. 1), then position vector at the $l^{\text {th }}$ sensor is given as $\mathbf{r}_{l}=(l-1) d \mathbf{a}_{x}$. Consider the case of a source moving with velocity $v$ and having a component along the direction of the line joining the origin and impinging angle $\theta$ at the $l^{\text {th }}$ sensor having a constant response $y_{l}(\theta)$. Then, $\mathbf{k}=\cos \theta \mathbf{a}_{x}+\sin \theta \mathbf{a}_{y}$ and output of the sensor is given as:

$$
\mathrm{x}\left(\mathbf{r}_{l}, t\right)=y_{l}(\theta) s(t) \operatorname{Re}\left[e^{j\left(\omega_{o} t-k(l-1) d \cos \theta\right.}\right]
$$

Also, $\omega_{o}$ will get shifted according to Doppler's classical shift formulae i.e. $\omega=\omega_{o}(1-v / c)$

Then, the output of $l^{\text {th }}$ sensor after dropping the term $e^{j \omega_{o} t}$ gets modified to:

$$
x\left(\mathbf{r}_{l}, t\right)=y_{l}(\theta) s(t) e^{-j \omega v t / c} e^{-j k(l-1) d \cos \theta}
$$

In case of $L$ sensors ULA, having same response for all sensors i.e. $y_{l}(\theta)=y(\theta)$, the output can be represented as a vector

$$
\mathbf{x}(t)=s(t) e^{-j \omega v t / c} \mathbf{a}(\theta)
$$

where, $\quad \mathbf{x}(t)=\left[\begin{array}{lllll}x_{1}(t) & x_{2}(t) & . & x_{L}(t)\end{array}\right] \quad$ and $\mathbf{a}_{\theta}=y(\theta)\left[\begin{array}{llll}1 & e^{-j k d \cos \theta}\end{array} . \quad e^{-j(L-1) k d \cos \theta}\right]$ which is also called steering vector. Consider the case of $p$ sources each located at an angle $\theta_{p}$. If the medium is considered to be linear then the output of each sensor can be written as:

$$
\begin{aligned}
x_{L}(t)= & s_{1}(t) y\left(\theta_{1}\right) e^{-j v_{1} \omega t / c} e^{-j k(L-1) d \cos \theta_{1}} \\
& +s_{2}(t) y\left(\theta_{2}\right) e^{-j v_{1} \omega t / c} e^{-j k(L-1) d \cos \theta_{2}} \\
& +s_{p}(t) y\left(\theta_{p}\right) e^{-j v_{1} \omega t / c} e^{-j k(L-1) d \cos \theta_{p}}
\end{aligned}
$$

If the sensor's response is same for every source i.e. $y\left(\theta_{1}\right)=y\left(\theta_{2}\right)=y\left(\theta_{p}\right)=y(\theta)$ then the above equation can be written as:

$$
\begin{array}{r}
{\left[\begin{array}{c}
x_{1}(t) \\
x_{2}(t) \\
\cdot \\
x_{L}(t)
\end{array}\right]=y(\theta)\left[\begin{array}{cccc}
1 & 1 & \cdot & 1 \\
e^{-j k d c o s} \theta_{1} & e^{-j k c \cos \theta_{2}} & \cdot & e^{-j k c \cos \theta_{p}} \\
\cdot & \cdot & \cdot & \cdot \\
e^{-j k(L-1) \cos \theta_{1}} & e^{-j k(L-1) \cos \theta_{2}} & \cdot & e^{-j k(L-1) \cos \theta_{p}}
\end{array}\right] \times} \\
\\
{\left[\begin{array}{c}
s_{1}(t) e^{-j \omega v_{1} t / c} \\
s_{2}(t) e^{-j \omega v_{2} t / c} \\
\cdot \\
s_{p}(t) e^{-j \omega v_{p} t / c}
\end{array}\right]}
\end{array}
$$

It can be represented as:

$$
\mathbf{x}(t)=\mathbf{A}(\theta) \mathbf{s}_{v}(t)
$$

Taking sensor's noise into consideration:

$$
\mathbf{x}(t)=\mathbf{A}(\theta) \mathbf{s}_{v}(t)+\mathbf{n}(t)
$$


If the sensor's output is sampled at $t=m \delta$ where $m=0,1 \ldots . . . k-1$ then:

$$
\mathbf{x}(m \delta)=\mathbf{A}(\theta) \mathbf{s}_{v}(m \delta)+\mathbf{n}(m \delta)
$$

From above equation the output for the $l^{\text {th }}$ sensor can be written as:

$$
x_{l}(m \delta)=\sum_{n=1}^{p} s_{n}(m \delta) e^{-j(l-1) d \cos \theta} \cdot e^{j \omega m \delta v_{n} / c}
$$

If $\mathrm{s}_{n}(m \delta)=s_{n}$, the above equation can be rewritten as:

$$
\mathbf{X}_{l}=\sum_{n=1}^{p} s_{n} e\left(\theta_{n}\right) \otimes \mathbf{f}\left(v_{n}\right)+\mathbf{w}_{\mathbf{l}}
$$

here $\mathbf{X}_{l}$ represents the time samples of the sensor output $x_{l}$.

Also

$$
e\left(\theta_{n}\right)=\left[\mathrm{e}^{-j k(l-1) \cos \theta_{n}}\right]
$$

and

$$
\mathbf{f}\left(v_{n}\right)=\left[\begin{array}{l}
1 \\
e^{-j \delta v_{n} / c} \\
e^{-j 2 \delta v_{n} / c} \\
\cdot \\
e^{-j(k-1) \delta v_{n} / c}
\end{array}\right]
$$

This concept can be extended to all the sensors and the resulting output can be written as:

$$
\mathbf{X}=\sum_{n=1}^{p} s_{n} \mathbf{e}\left(\theta_{n}\right) \otimes \mathbf{f}\left(v_{n}\right)+\mathbf{w}
$$

here

$$
\mathbf{e}\left(\theta_{n}\right)=\left[\begin{array}{lllll}
1 & e^{-j k d \cos \theta_{n}} & \cdot & e^{-j(L-1) k d \cos \theta_{n}}
\end{array}\right]^{T}
$$

The above equation can also be written as:

$$
\mathbf{X}=\mathbf{S}\left[\mathbf{e}\left(\theta_{1}\right) \otimes \mathbf{f}\left(v_{1}\right) \quad \mathbf{e}\left(\theta_{2}\right) \otimes \mathbf{f}\left(v_{2}\right) \quad . \quad . \quad \mathbf{e}\left(\theta_{p}\right) \otimes \mathbf{f}\left(v_{p}\right)\right]+\mathbf{W}
$$
where

$$
\mathbf{S}=\left[\begin{array}{lllll}
s_{1} & s_{2} & \cdot & \cdot & s_{p}
\end{array}\right]^{T}
$$

We define

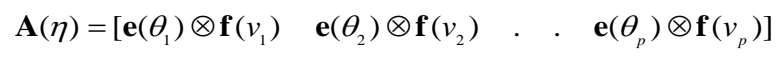

here $\mathbf{S}$ is $p \times 1$ and $\mathbf{A}(\eta)$ is $L k \times p$ matrix. Using above representations, the final form of the data model is:

$$
\mathbf{X}=\mathbf{A}(\eta) \mathbf{S}+\mathbf{W}
$$

\section{PARAMETER ESTIMATION}

In the data model signal waveforms are assumed to be deterministic but unknown and noise is modelled as i.i.d. spatio-temporal white Gaussian random process with constant variance i.e. $\mathbf{W}=\sigma^{2} \mathbf{I}_{L k \times 1}$. As a result $\mathbf{X}$ is also a Gaussian random process with mean $\mathbf{A}(\eta) \mathbf{S}$ and variance $\sigma^{2} \mathbf{I}$. The PDF of a Gaussian random variable is given by:

$$
p(x)=\frac{1}{\sqrt{2 \pi \sigma^{2}}} e^{-(x-\mu)^{2} / 2 \sigma^{2}}
$$

Here $\mu$ and $\sigma^{2}$ represent mean and variance of the random variable. Using (12), the PDF of observation vector $\mathbf{X}$ is given by:

$$
Y=C e^{-\|\mathbf{X}-\mathbf{A}(\eta) \mathbf{S}\|^{2} / 2 \sigma^{2}}
$$

where, $C$ is a constant and does not contribute to decision making. Taking log of both sides of (13), we get

$$
Z=-\frac{C}{2 \sigma^{2}}\|\mathbf{X}-\mathbf{A}(\eta) \mathbf{S}\|^{2}
$$

Neglecting the term $-\frac{C}{2 \sigma^{2}}$, we get PDF as:

$$
Y=\|\mathbf{X}-\mathbf{A}(\eta) \mathbf{S}\|^{2}
$$

The maximum-likelihood estimation rule dictates that (15) is to be minimized with respect to $\eta$. First, to obtain maximum-likelihood (ML) estimate of $S$, the derivative of (15) with respect to $S$ is set to zero. This will result in

$$
S^{\prime}=\left(A^{H} A\right)^{-1} A^{H} X
$$

where $A$ is a function of $\eta$. Using (15) and (16), we get

$$
Y=\|X-P(\eta) X\|^{2}
$$


where $P(\eta)=A\left(A^{H} A\right)^{-1} A^{H}$. The above expression can be rewritten as:

$$
Y=\left(\|X\|^{2}-\|P(\eta) X\|^{2}\right)
$$

Minimizing the above expression implies maximizing $\|P(\eta) X\|^{2}$. So, the ML estimate of parameter $\eta$ is given by:

$$
\eta^{\prime}=\operatorname{Arg} \max _{\eta}\|P(\eta) X\|^{2}
$$

The MLE estimate can be determined by exploiting the non-linear optimization algorithms. The basic principle behind non-linear optimization is to find the optimal parameters so that log-likelihood function is maximized [14]. This can be achieved by dividing the multi-dimensional parameter space into smaller sub-sets and applying trial and error approach such that for next iteration parameters value is modified so that it leads to better performance than that for previous parameters values.

\section{SimUlations}

Many simulations have been conducted to explore the various aspects of above discussed algorithm. The numbers of sensors on the array were chosen to be five with uniform spacing between them. Also, the centre frequency was chosen as $100^{*} c$, where $c$ the velocity of light. The sampling interval $\delta$ was chosen to be equal to the wavelength $(\lambda)$ of the signal. The spacing $(d)$ between sensors was chosen to be $\lambda / 10$. Also, the numbers of targets were chosen to be two and it was also assumed that numbers of targets are known in advance. The noise was assumed to be additive white Gaussian noise with zero mean and unit variance. Also, the velocity of targets was restricted between $1 / 50$ to $1 / 10 \mathrm{~m} / \mathrm{sec}$ i.e. $v=\left[\frac{1}{50}, \frac{1}{10}\right]$ $\mathrm{m} / \mathrm{sec}$, however, no restriction was made in the direction of targets i.e. $\theta=[0,2 \pi]$ radians.

\section{A. Direction Estimations of Targets}

Two targets were located at $\pi / 4$ and $\pi / 2$ radians respectively and were of unequal power. Also, the signal to noise ratio (SNR) for each target was $10 \mathrm{~dB}$ and 51 iterations were conducted, one for estimation under the ideal condition and other 50 for estimation when the noise was present. In each trial noise was different. Estimations are illustrated in Fig.2 and Fig.3.

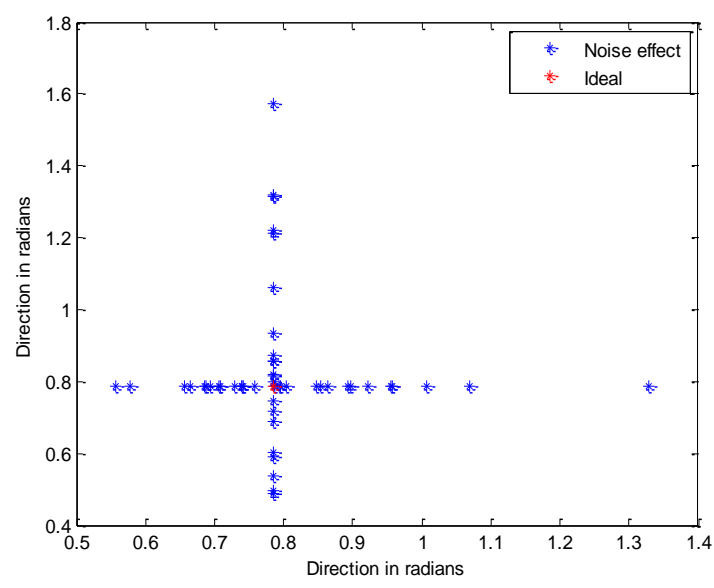

Fig.2. Direction estimation of the first target at 10dB

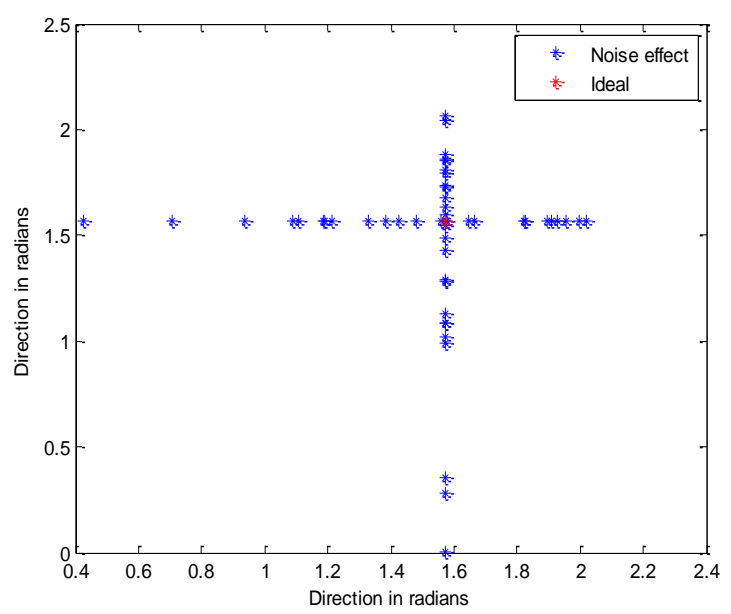

Fig.3. Direction estimation of the second target at 10dB

\section{B. Velocity Estimations of the Targets}

Targets were having a velocity of $1 / 40$ and $1 / 20 \mathrm{~m} / \mathrm{s}$. Other conditions were same as for direction estimation. Estimations are illustrated in Fig.4 and Fig.5.

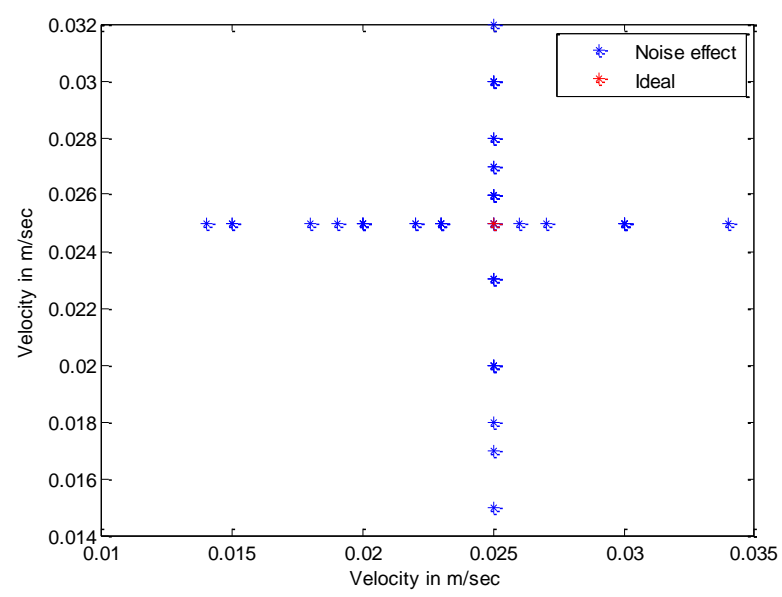

Fig.4. Velocity estimation of the first target 


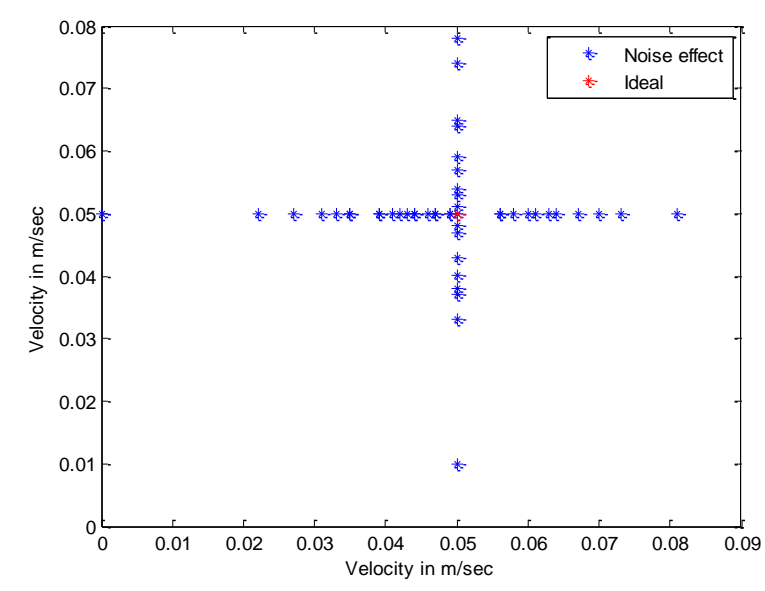

Fig.5. Velocity estimation of the second target

Another 51 iterations were made for these two targets with SNR of $20 \mathrm{~dB}$. Results are shown in Fig.6, Fig.7, Fig.8 and Fig.9.

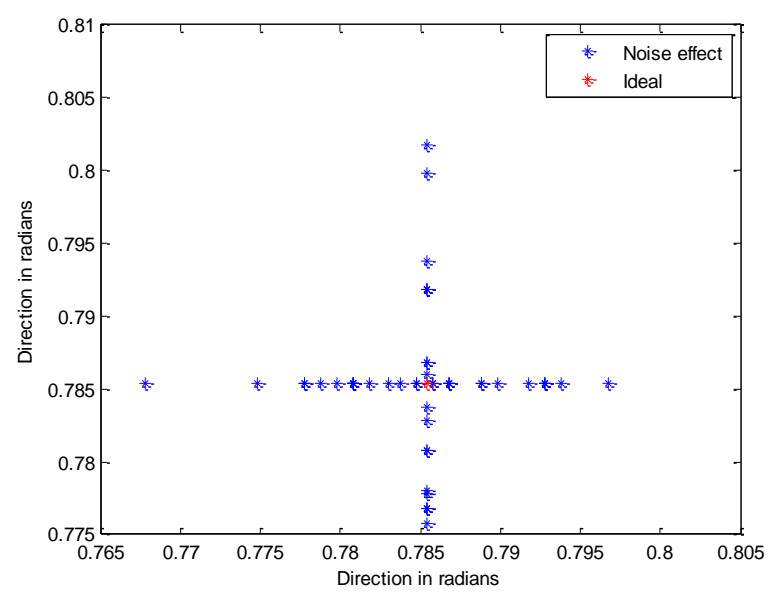

Fig.6. Direction estimation of the first target at $20 \mathrm{~dB}$

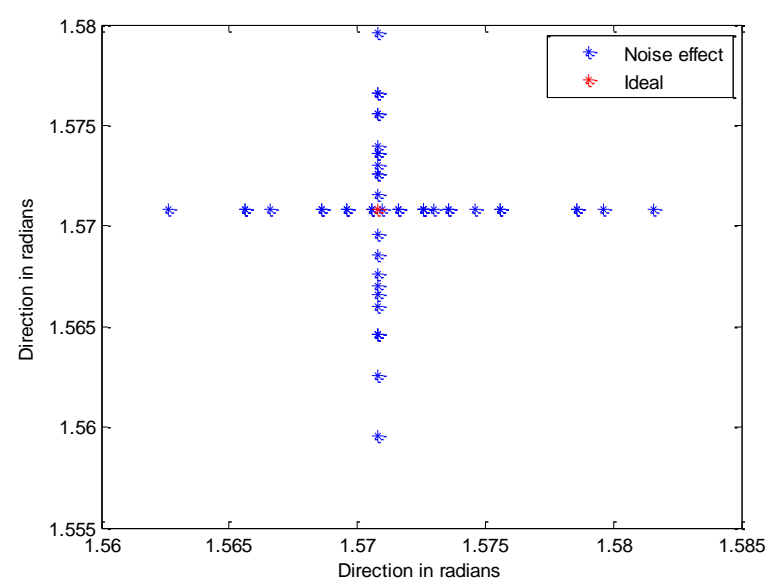

Fig.7. Direction estimation of the second target at $20 \mathrm{~dB}$

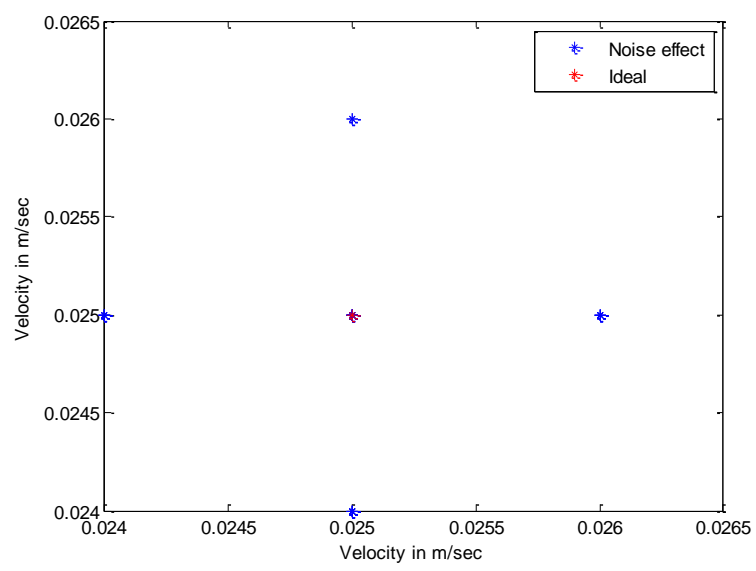

Fig.8. Velocity estimation of the first target at $20 \mathrm{~dB}$

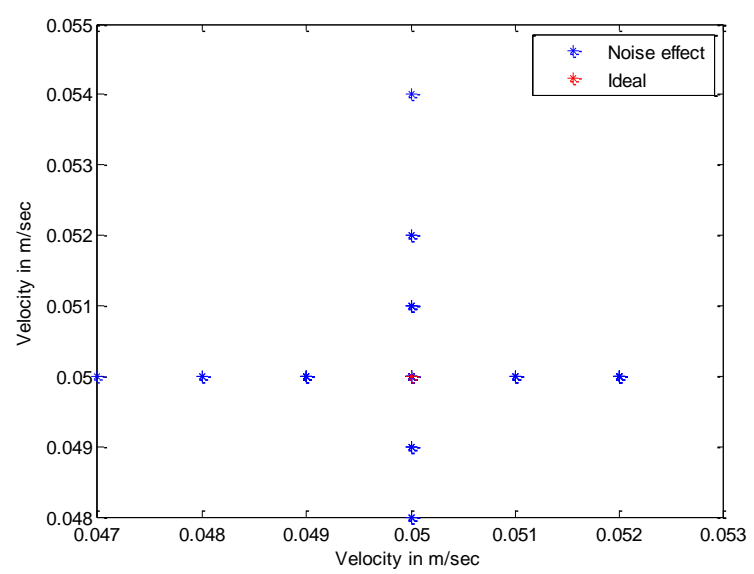

Fig.9. Velocity estimation of the second target at $20 \mathrm{~dB}$

Simulations depict the validity of the algorithm for ideal case and to a greater extent for practical scenario as well (presence of noise). Also, it can be observed that effect of noise is less prominent in case of velocity estimation as compared with the case of direction estimation. Further, the average error in estimations for different SNR is summarized in table 1.

Table 1. Average error in parameters estimation

\begin{tabular}{|c|c|c|}
\hline SNR & $\begin{array}{c}\text { Error in direction } \\
\text { estimation }\end{array}$ & $\begin{array}{c}\text { Error in velocity } \\
\text { estimation }\end{array}$ \\
\hline $10 \mathrm{~dB}$ & .65 & .034 \\
\hline $20 \mathrm{~dB}$ & .011 & .033 \\
\hline
\end{tabular}

\section{Effect of enhanced SNR on estimation error}

Fig.10 and Fig.11 depict the effect of SNR on error in estimations. 


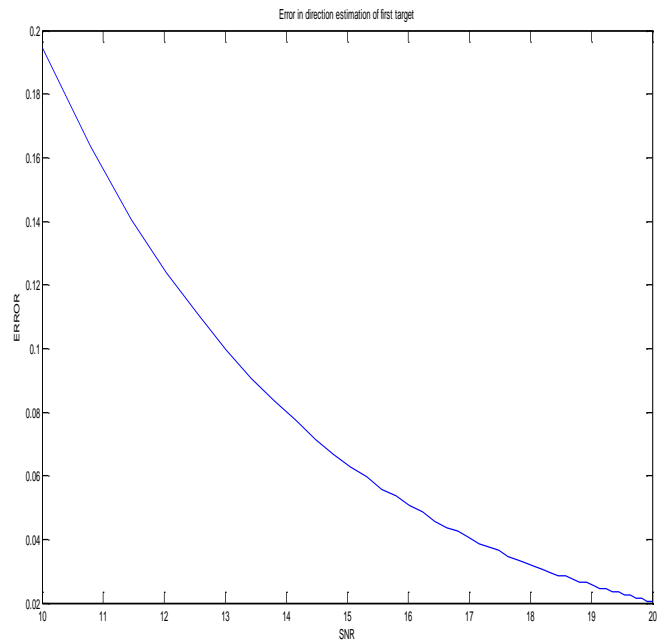

Fig.10. Effect on direction estimation

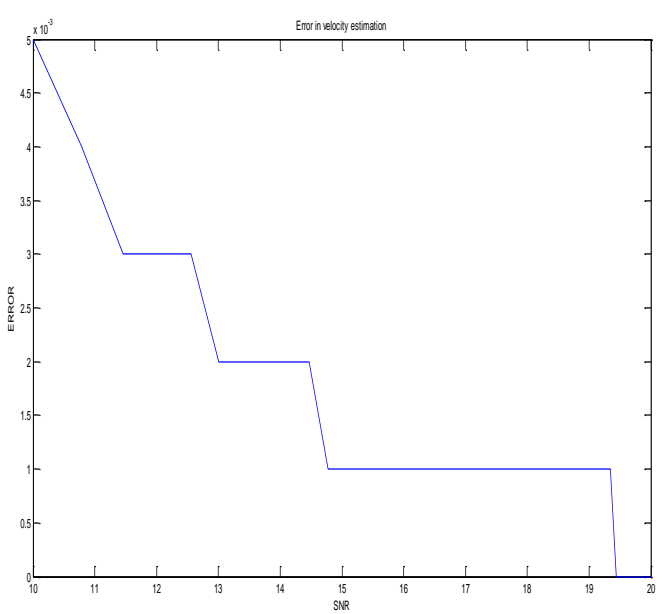

Fig.11. Effect on velocity estimation

It is observed from Fig.10 and Fig.11 that performance of the algorithm is improving drastically with improvement in SNR and theoretically at very high SNR the algorithm will achieve noise immunity.

\section{MUSIC ESTIMATOR}

For the sake of completeness and for demonstrating the pragmatic aspect of the developed algorithm, a comparison with MUSIC estimator is made in this section. MUSIC was introduced as a most effective DOA estimation technique which proceeds by decomposition of covariance matrix and forming a spectrum using steering vector. MUSIC works well for ideal case and uncorrelated noise but fails for the correlated noise.

Reconsider the data model

$$
\mathbf{x}(t)=\mathbf{A}(\theta) \mathbf{s}_{v}(t)+\mathbf{n}(t)
$$

We define spatial-covariance matrix as:

$$
\begin{aligned}
& \mathbf{R}=E\left\{\mathbf{x}(t) \mathbf{x}^{H}(t)\right\} \\
& \mathbf{R}=\mathbf{A}(\theta) E\left\{\mathbf{s}_{v}(t) \mathbf{s}_{v}{ }^{H}(t)\right\} \mathbf{A}^{H}+E\left\{\mathbf{n}(t) \mathbf{n}^{H}(t)\right\}
\end{aligned}
$$

If noise is assumed to be spatio-temporal white with common variance $\sigma^{2}$ then

$$
E\left\{\mathbf{n}(t) \mathbf{n}^{H}(t)\right\}=\sigma^{2} \mathbf{I}
$$

and if $\mathbf{P}=E\left\{\mathbf{s}_{v}(t) \mathbf{s}_{v}{ }^{H}(t)\right\}$ then $\mathbf{R}$ will become

$$
\mathbf{R}=\mathbf{A}(\theta) \mathbf{P A}^{H}(\theta)+\sigma^{2} \mathbf{I}
$$

$\mathbf{R}$ can be factored as $\mathbf{R}=U \Lambda U^{H}$ with $\Lambda=\operatorname{diag}\left[\begin{array}{llll}\lambda_{1} & \lambda_{2} & . & \lambda_{L}\end{array}\right]$ is a diagonal matrix of eigen values $\lambda_{1}, \lambda_{2}, \ldots \lambda_{L}$ and $U$ is a unitary matrix having eigen vectors corresponding to eigen values $\lambda_{1}, \lambda_{2}, \ldots \lambda_{L}$. $\mathbf{R}$ can also be written as

$$
\mathbf{R}=U_{s} \Lambda_{n} U_{s}{ }^{H}+U_{n} \Lambda_{n} U_{n}{ }^{H}
$$

with $\Lambda_{n}=\sigma^{2} \mathbf{I}$. In practise, an estimate of $\mathbf{R}$ is found as

$$
\mathbf{R}=\frac{1}{k} \sum_{t=1}^{k} \mathbf{x}(t) \mathbf{x}^{H}(t)
$$

Hence,

$$
\mathbf{R}_{s}=\tilde{U}_{s} \tilde{\Lambda}_{s} \tilde{U}_{s}^{H}+\tilde{U}_{n} \tilde{\Lambda}_{n} \tilde{U}_{n}^{H}
$$

The projector operators onto signal subspace are defined as:

$$
\tilde{\Pi}=\tilde{U}_{s} \tilde{U}_{s}^{H}=\tilde{\mathbf{A}}(\boldsymbol{\theta})\left(\tilde{\mathbf{A}}(\theta)^{H} \tilde{\mathbf{A}}(\theta)\right)^{-1} \tilde{\mathbf{A}}(\theta)^{H}
$$

Similarly, projection onto noise signal subspace is defined as:

$$
\tilde{\Pi}^{\perp}=\tilde{U}_{n} \tilde{U}_{n}^{H}=\mathbf{I}-\tilde{\mathbf{A}}(\boldsymbol{\theta})\left(\tilde{\mathbf{A}}(\theta)^{H} \tilde{\mathbf{A}}(\theta)\right)^{-1} \tilde{\mathbf{A}}(\theta)^{H}
$$

The MUSIC spatial spectrum is then defined as:

$$
P_{M}(\theta)=\frac{\mathbf{a}^{H}(\theta) \mathbf{a}(\theta)}{\mathbf{a}^{H}(\theta) \tilde{\Pi}^{\perp} \mathbf{a}(\theta)}
$$

Where, $\mathbf{a}(\theta)$ is steering vector.

$P_{M}(\theta)$ is not a true spectrum but instead gives a peak at the point of direction of arrival (DOA).

A. Simulations 
Several simulations were carried out to analyse the performance of MUSIC algorithm and to compare it with the developed algorithm for the case of (DOA) estimation. Two sources were chosen in the far field of sensors array which were located at $\pi / 3$ and $\pi / 2$ radians. Also, the numbers of sensors were chosen to be five. Few simulations were carried under ideal conditions (absence of noise) and then under uncorrelated noise conditions. Simulations were also carried for correlated noise with different SNR.

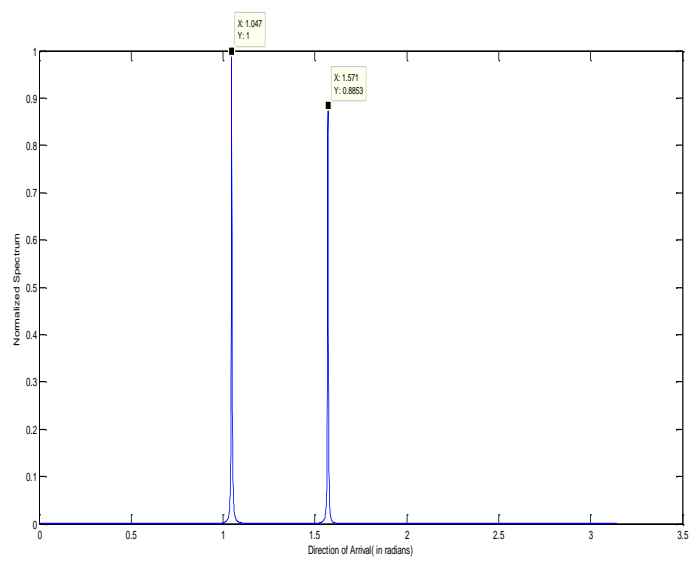

Fig.12. Ideal estimation

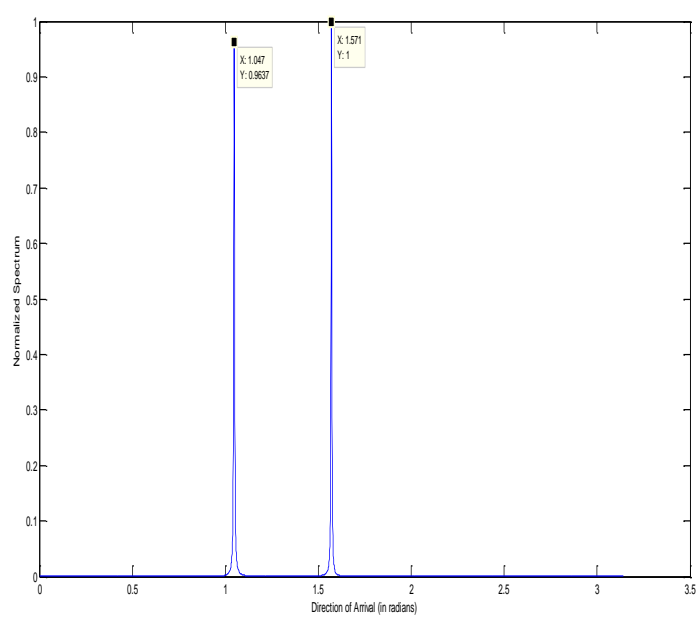

Fig.13. Estimation under uncorrelated noise

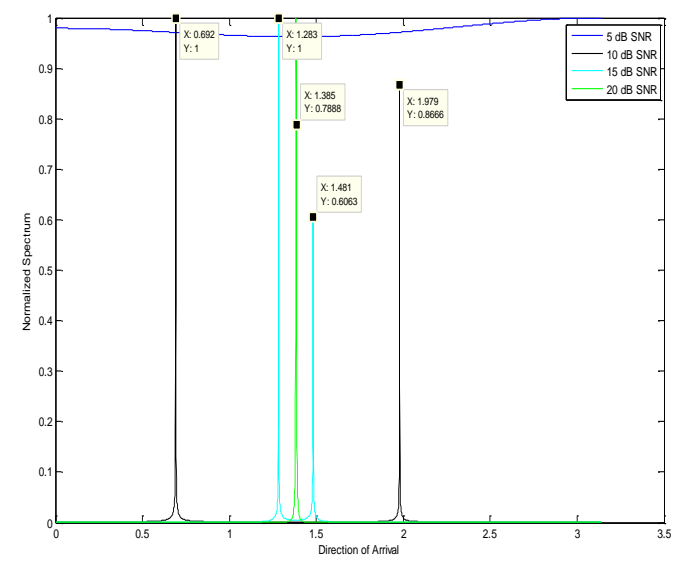

Fig.14. Estimation under correlated noise for different SNR

It can be inference from the simulations that MUSIC and proposed algorithm are contrasting for the case of correlated noise. The performance of MUSIC is compromised drastically for this case but for ideal case and uncorrelated noise MUSIC is always way ahead of the proposed algorithm considering its affordable computational complexity. Table 2 further highlights the key differences between MUSIC and proposed algorithm.

Table 2. Comparison of proposed algorithm and MUSIC

\begin{tabular}{|c|c|c|}
\hline Parameter & Proposed algorithm & MUSIC \\
\hline Principle & Parametric approach & $\begin{array}{c}\text { Spectral } \\
\text { decomposition }\end{array}$ \\
\hline $\begin{array}{c}\text { Performance under } \\
\text { uncorrelated noise }\end{array}$ & Good & Good \\
\hline $\begin{array}{c}\text { Performance under } \\
\text { correlated noise }\end{array}$ & Good & Degraded \\
\hline $\begin{array}{c}\text { Computational } \\
\text { complexity }\end{array}$ & High & Less \\
\hline Consistency & Yes & Yes \\
\hline $\begin{array}{c}\text { Effect of enhanced } \\
\text { SNR }\end{array}$ & $\begin{array}{c}\text { Improved } \\
\text { performance }\end{array}$ & $\begin{array}{c}\text { Improved } \\
\text { performance }\end{array}$ \\
\hline
\end{tabular}

\section{CRAMER-RAO Bound CALCULATION}

The purpose of an estimation algorithm is to map the message $S_{i}$ to $\tilde{S}_{i}$. The adjacency of $S_{i}$ and $\tilde{S}_{i}$ can be used to specify the integrity of the receivers. For a probabilistic problem the PDF and variance are used to measure the deviation from ideal estimater. A suitable measure of the performance for an unbiased estimation [15] is Cramer-Rao bound. The Cramer-Rao bound [16] is given as: 


$$
\operatorname{var}\left(S_{i}(\mathbf{x}) \mid S_{i}\right) \geq\left(E\left(\left[\frac{\partial}{\partial S_{i}} \ln p\left(\mathbf{x} \mid S_{i}\right)\right]^{2}\right)\right)^{-1}
$$

where $\mathbf{X}$ is the observation vector. The unbiased estimator for which Cramer-Rao bound is satisfied with equality is called efficient estimator. In this section we proceed with the Cramer-Rao bound calculation for the developed algorithm.

Again, consider the data model

$$
\mathbf{X}=\mathbf{A}(\eta) \mathbf{S}+\mathbf{W}
$$

We define

$$
E(\boldsymbol{\theta}, \mathbf{v})=\operatorname{col}\left[\mathbf{e}\left(\boldsymbol{\theta}_{\boldsymbol{\alpha}}\right) \otimes \mathbf{f}\left(\mathbf{v}_{\boldsymbol{\alpha}}\right)\right], \mathbf{1} \leq \boldsymbol{\alpha} \leq \mathbf{m}
$$

Using (13), we get

$$
\begin{aligned}
-\log p(\mathbf{x} \mid S)=\frac{1}{\sigma^{2}}\|\mathbf{x}-\mathbf{E S}\|^{2} \\
L=\frac{1}{\sigma^{2}}\|\mathbf{x}-\mathbf{E S}\|^{2} \\
\sigma^{2} L=\|\mathbf{x}-\mathbf{E S}\|^{2} \\
=\left(\mathbf{x}^{\mathbf{H}}-\mathbf{S}^{\mathbf{H}} \mathbf{E}^{\mathbf{H}}\right)(\mathbf{x}-\mathbf{E S}) \\
=\|\mathbf{x}\|^{2}-\mathbf{2} \mathfrak{R}\left(\mathbf{S}^{\mathbf{H}} \mathbf{E}^{\mathbf{H}} \mathbf{x}\right)+\mathbf{S}^{\mathbf{H}} \mathbf{E}^{\mathbf{H}} \mathbf{E S} \\
=\|\mathbf{x}\|^{2}-2 \mathfrak{R}\left(\mathbf{S}_{R}-j \mathbf{S}_{I}\right)^{T} \mathbf{E}^{H} \mathbf{x}+. . \\
. .\left(\mathbf{S}_{R}-j \mathbf{S}_{I}\right)^{T}\left(\mathbf{E}^{H} \mathbf{E}\right)\left(\mathbf{S}_{R}+j \mathbf{S}_{I}\right)
\end{aligned}
$$

where we have assumed $\mathbf{S}=\mathbf{S}_{R}+j \mathbf{S}_{I}$.

Now, we calculate the following derivatives:

$$
\begin{aligned}
& \frac{\partial^{2}\left(\sigma^{2} L\right)}{\partial \mathbf{S}_{R} \partial \mathbf{S}_{R}^{T}}=2 \mathbf{E}^{T} \mathbf{E}=\frac{\partial^{2}\left(\sigma^{2} L\right)}{\partial \mathbf{S}_{I} \partial \mathbf{S}_{I}^{T}} \\
& \frac{\partial^{2}\left(\sigma^{2} L\right)}{\partial \mathbf{S}_{R} \partial \mathbf{S}_{I}^{T}}=j\left(\mathbf{E}^{H} \mathbf{E}-\left(\mathbf{E}^{H} \mathbf{E}\right)^{T}\right)=-2 \mathfrak{J}\left(\mathbf{E}^{H} \mathbf{E}\right) \\
& \frac{\partial^{2}\left(\sigma^{2} L\right)}{\partial \mathbf{S}_{I} \partial \mathbf{S}_{R}^{T}}=-2 \Im\left(\mathbf{E}^{H} \mathbf{E}\right)^{T}=2 \Im\left(\mathbf{E}^{H} \mathbf{E}\right) \\
& \frac{\partial^{2}\left(\sigma^{2} L\right)}{\partial \theta_{\alpha} \partial \theta_{\beta}}=-2 \Re\left(\mathbf{S}^{H} \frac{\partial^{2} \mathbf{E}^{H}}{\partial \theta_{\alpha} \partial \theta_{\beta}} \mathbf{x}\right)+\mathbf{S}^{H} \frac{\partial^{2} \mathbf{E}^{H} \mathbf{E}}{\partial \theta_{\alpha} \partial \theta_{\beta}} \mathbf{S} \\
& \frac{\partial^{2}\left(\sigma^{2} L\right)}{\partial v_{\alpha} \partial v_{\beta}}=-2 \Re\left(\mathbf{S}^{H} \frac{\partial^{2} \mathbf{E}^{H}}{\partial v_{\alpha} \partial v_{\beta}} \mathbf{x}\right)+\mathbf{S}^{H} \frac{\partial^{2} \mathbf{E}^{H} \mathbf{E}}{\partial v_{\alpha} \partial v_{\beta}} \mathbf{S} \\
& \frac{\partial^{2}\left(\sigma^{2} L\right)}{\partial \theta_{\alpha} \partial v_{\beta}}=-2 \Re\left(\mathbf{S}^{H} \frac{\partial^{2} \mathbf{E}^{H}}{\partial \theta_{\alpha} \partial v_{\beta}} \mathbf{x}\right)+\mathbf{S}^{H} \frac{\partial^{2} \mathbf{E}^{H} \mathbf{E}}{\partial \theta_{\alpha} \partial v_{\beta}} \mathbf{S}^{H} \\
& \frac{\partial^{2}\left(\sigma^{2} L\right)}{\partial \mathbf{S}_{R} \partial \theta_{\alpha}}=-2 \mathfrak{R}\left(\frac{\partial^{2} \mathbf{E}^{H}}{\partial \theta_{\alpha}} \mathbf{x}\right)+2 \Re \frac{\partial\left(\mathbf{E}^{H} \mathbf{E}\right)}{\partial \theta_{\alpha}} \mathbf{S}_{R}-2 \mathfrak{J} \frac{\partial\left(\mathbf{e}^{H} \mathbf{E}\right)}{\partial \theta_{\alpha}} \mathbf{S}_{I} \\
& \frac{\partial^{2}\left(\sigma^{2} L\right)}{\partial \mathbf{S}_{I} \partial \theta_{\alpha}}=-2 \mathfrak{I}\left(\frac{\partial^{2} \mathbf{E}^{H}}{\partial \theta_{\alpha}} \mathbf{x}\right)+2 \Re \frac{\partial\left(\mathbf{E}^{H} \mathbf{E}\right)}{\partial \theta_{\alpha}} \mathbf{S}_{R}+2 \mathfrak{I} \frac{\partial\left(\mathbf{e}^{H} \mathbf{E}\right)}{\partial \theta_{\alpha}} \mathbf{S}_{R}
\end{aligned}
$$

and similarly, we can calculate

$$
\frac{\partial^{2}\left(\sigma^{2} L\right)}{\partial \mathbf{S}_{R} \partial v_{\alpha}} \text { and } \frac{\partial^{2}\left(\sigma^{2} L\right)}{\partial \mathbf{S}_{I} \partial v_{\alpha}}
$$

Fisher-Information matrix [17] is given as:

$$
\sigma^{2} \mathbf{J}(\varphi)=\sigma^{2} E\left(\frac{\sigma^{2} L}{\partial \varphi \partial \varphi^{T}}\right)
$$

This matrix has to be written for parameter $\varphi$ i.e.

$$
\begin{aligned}
& \mathbf{J}_{\theta \theta}, \mathbf{J}_{\theta v}, \mathbf{J}_{\theta S_{R}}, \mathbf{J}_{\theta S_{I}}, \mathbf{J}_{v \theta}, \mathbf{J}_{v \theta}, \mathbf{J}_{v S_{R}}, \mathbf{J}_{v S_{I}}, \mathbf{J}_{S_{R} \theta}, \mathbf{J}_{S_{R} v}, \\
& \mathbf{J}_{S_{R} S_{R}}, \mathbf{J}_{S_{R} S_{I}}, \mathbf{J}_{S_{I} \theta}, \mathbf{J}_{S_{I} v}, \mathbf{J}_{S_{I} S_{R}}, \mathbf{J}_{S_{I} S_{I}}
\end{aligned}
$$

After calculating all these terms, the results are substituted in (26) which will provide the Cramer-Rao bound of the developed algorithm.

\section{CONCLUSION}

We proposed and investigated an algorithm based on MLE and also contrasted it with MUSIC algorithm. During simulations, it was found that for the case of uncorrelated noise both the algorithms had the same performance, MUSIC, in addition, had the advantage of low computational complexity but for the case of correlated noise MUSIC algorithm had a degraded performance while ML based algorithm continued to work satisfactorily. However, the price paid for this advantage was increased computational complexity as ML uses multidimensional search to find the estimates. For the case of correlated noise, even at high SNR, the performance of MUSIC estimator was not satisfactory while ML based algorithm was showing a significant performance improvement with SNR. Also, the improvement for velocity estimation was more pronounced than for DOA estimation.

\section{REFERENCES}

[1] Hamid Karim and Mats Viberg, "Two Decade of Array Signal Processing,"IEEE Signal Processing Magazine, July 1996.

[2] Schmidt, R.O, "Multiple Emitter Location and Signal Parameter Estimation," IEEE Trans. Antennas Propagation, Vol. AP-34 (March 1986), pp.276-280.

[3] Belouchrani, A.; Amin, M.G., "Time-frequency MUSIC," Signal Processing Letters, IEEE, vol.6, no.5, pp.109,110, May 1999.

[4] Paulraj, A.; Roy, R.; Kailath, T., "A subspace rotation approach to signal parameter estimation," Proceedings of the IEEE, vol.74, no.7, pp.1044,1046, July 1986.

[5] Roy, R.; Kailath, T., "ESPRIT-estimation of signal parameters via rotational invariance techniques," Acoustics, Speech and Signal Processing, IEEE Transactions on, vol.37, no.7, pp.984,995, Jul 1989.

[6] GIRD Systems, Inc. 310 Terrace Ave. Cincinnati, Ohio 45220, "An Introduction to MUSIC and ESPRIT". 
[7] Lavate, T.B.; Kokate, V.K.; Sapkal, A.M., "Performance Analysis of MUSIC and ESPRIT DOA Estimation Algorithms for Adaptive Array Smart Antenna in Mobile Communication," Computer and Network Technology (ICCNT), 2010 Second International Conference on, vol., no., pp.308,311, 23-25 April 2010.

[8] H.L. Van Trees, Detection, Estimation, and Modulation Theory, New York, Wiley 1971.

[9] Myung, In Jae. "Tutorial on maximum likelihood estimation," Journal of mathematical Psychology 47.1 (2003): 90-100.

[10] S.K. Gehlot, Ravendra Singh, "Trajectory Estimation of a moving charged particle: An application of least square estimation (LSE) approach", IEEE, Medcom 2014, pp 262-265, Nov 2014.

[11] Saea A. Van De Geer, Least Squares Estimation, Encyclopaedia of Statistics in Behavioral Science, Volume 2, pp. 10411045.

[12] C. Radhakrishnan Rao. Linear Statistical Inference and Its Applications,John Wiley Sons 1965.

[13] H. L. Van Trees, "Optimum array processing - Part IV of detection, estimation, and modulation theory", John Wiley, 2002

[14] Gholami, M.R.; Gezici, S.; Strom, E.G.; Rydstrom, M., "Positioning algorithms for cooperative networks in the presence of an unknown turn-around time," Signal Processing Advances in Wireless Communications (SPAWC), 2011 IEEE 12th International Workshop on, vol., no., pp.166,170, 26-29 June 2011.

[15] Voinov, Vassily ; Nikulin, Mikhail (1993). Unbiased estimators and their applications. 1: Univariate case. Dordrect: Kluwer Academic Publishers. ISBN 0-7923-2382-3.

[16] Zachariah, D.; Stoica, P., "Cramer-Rao Bound Analog of Bayes' Rule [Lecture Notes]," Signal Processing Magazine, IEEE , vol.32, no.2, pp.164,168, March 2015.

[17] Frieden, B. Roy. Science from Fisher information: a unification. Cambridge University Press, 2004.

\section{Authors' Profiles}

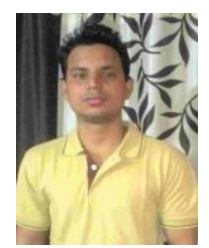

Shiv Gehlot received the B.Tech. degree in Electronics \& Communication engineering from Uttar Pradesh Technical University in 2011. He completed his M.Tech. degree in Signal Processing at Netaji Subash Institute of Technology in 2013. His present research areas include Statistical Signal Processing and Digital Image Processing. Presently, he is working as an Assistant Professor in the department of Electronics \& Communication Engineering at Noida Institute of Engineering \& Technology, Greater Noida.

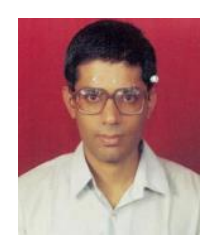

Prof. Harish Parthasaraty received B.Tech in 1990 (from Indian Institute of Technology, Kanpur, India) and Ph. D. (from India Institute of Technology, Delhi, India) in 1994, both in Electrical Engineering. Presently, he is working as Professor in the division of Electronics \& Communication Engineering at Netaji Subash Institute of Technology, New-Delhi. His teaching and research interests are in the areas of circuits and systems, signal processing, stochastic nonlinear filters, electromagnetics and group representations and he has published 30 papers in various international journals of repute.

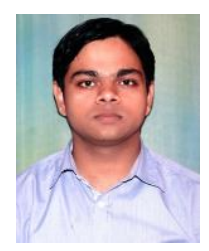

Ravendra Singh received B.Tech in Electronics \& Communication Engineering and M.Tech. in Signal Processing from Rajiv Gandhi Proudyogiki Vishwavidyalaya and Netaji Subash Institute of Technology respectively. Presently, he is working as an Assistant Professor in the department of Electronics \& Communication Engineering at Galgotias University, Greater Noida.

How to cite this paper: Shiv Gehlot, Harish Parthasarathy, Ravendra Singh,"Two-Dimensional Parameters Estimation", International Journal of Image, Graphics and Signal Processing(IJIGSP), Vol.8, No.9, pp.1-9, 2016.DOI: 10.5815/ijigsp.2016.09.01 